\title{
Modeling Orientation Selectivity Using a Neuromorphic Multi-Chip System
}

\author{
Elisabetta Chicca, Patrick Lichtsteiner, Tobias Delbruck, Giacomo Indiveri and Rodney J. Douglas \\ Institute of Neuroinformatics \\ University/ETH Zurich \\ Winterthurerstrasse 190, CH-8057 \\ Email: chicca@ini.phys.ethz.ch
}

\begin{abstract}
The growing interest in pulse-mode processing by neural networks is encouraging the development of hardware implementations of massively parallel, distributed networks of Integrate-and-Fire (I\&F) neurons. We have developed a reconfigurable multi-chip neuronal system for modeling feature selectivity and applied it to oriented visual stimuli. Our system comprises a temporally differentiating imager and a VLSI competitive network of neurons which use an asynchronous Address Event Representation (AER) for communication. Here we describe the overall system, and present experimental data demonstrating the effect of recurrent connectivity on the pulse-based orientation selectivity.
\end{abstract}

\section{INTRODUCTION}

Neuromorphic systems are composed of mixed ana$\log$ /digital VLSI devices that emulate biological systems for sensory processing. We propose a neuromorphic system that reproduces a specific functionality of neocortical processing modules: the processing of visual input by the neuronal circuits of the mammalian visual cortex. Despite significant differences in function across the various cortical areas, the pattern of neuronal connections within each area is remarkably similar. This regular structure suggests that the cortex may use a common core processing circuit, or canonical microcircuit, that can be tuned to perform specific tasks [1]. The canonical microcircuit, and its later extensions, emphasize the role of first order recurrent connections between cortical neurons. These recurrent connections between thresholded neurons support cooperative-competitive processing, in which networks of neurons participate collectively in the generation of an appropriate interpretation of their sensory input. The output of a given neuron depends dynamically on the activity of all neurons in the network. As a result, these networks are able to perform complicated non-linear operations, such as the winner-take-all function. The computational abilities arising from competition are especially useful for feature extraction and pattern classification problems. We are interested in capturing the principle of cooperative-competitive processing in a general purpose module that could be used to build complex VLSI perceptive systems.

In this paper we present a specific application, in which a VLSI recurrent network of spiking neurons, interfaced to a vision sensor, is used to implement orientation selectivity. Several theoretical recurrent models have been proposed to explain the origin of orientation selectivity in primary visual cortex and as a means to understand cortical circuitry and cortical computation (for a review see [2]). The pattern of connectivity described in these feed-back models is reflected in the local recurrent connections of our VLSI network of spiking neurons: similar orientation cooperate through lateral excitation and different orientations compete through global inhibition.

Several hardware models of orientation selectivity have been proposed in the past [3]-[7]. Our approach differs from those of [3], [5] in that our system decouples the sensing stage from the computational stage. In this way the computational stage can be more modular and more easily expanded. Also, our computation depends on the collective dynamics of a population of neurons, rather than the explicit implementation of a complex function for the receptive field, such as edge enhancement by high-pass filtering [4], or Gabor functions [3], [7]. Our approach is flexible, because it depends only on the connection pattern among many similar processing elements, the neurons. Thus, the computational part of the system is not explicitly designed for orientation selectivity. Instead, it models a more generic cortical module that can be applied to the detection of other features, and to other sensory modalities (e.g. audition).

The system we developed is an evolution of the one proposed by Liu et al. [6] in which neurons tuned to the same retinal position but different orientations are on the same chip. It differs from that of Liu et al. because it uses recurrent excitation in addition to recurrent inhibition and it models several orientation selective neurons generating a more realistic network in which neurons with similar preferred orientation cooperate, and neurons tuned to different orientations compete. As in [3], [4], [7] we use the Address Event Representation (AER) scheme for the communication between our neuromorphic VLSI chips.

\section{THE ORIENTATION SELECTIVITY SYSTEM COMPONENTS}

The system consists of two neuromorphic VLSI chips, a PCI-AER board [8] and supporting hardware (see Fig. 1). The neuromorphic chips are an address-event temporally differentiating imager (TMPDIFF chip) and a recurrent competitive network of integrate-and-fire neurons and dynamic synapses (IFWTA chip). The PCI-AER board is a custom device which provides a communication bridge between the AER and the 


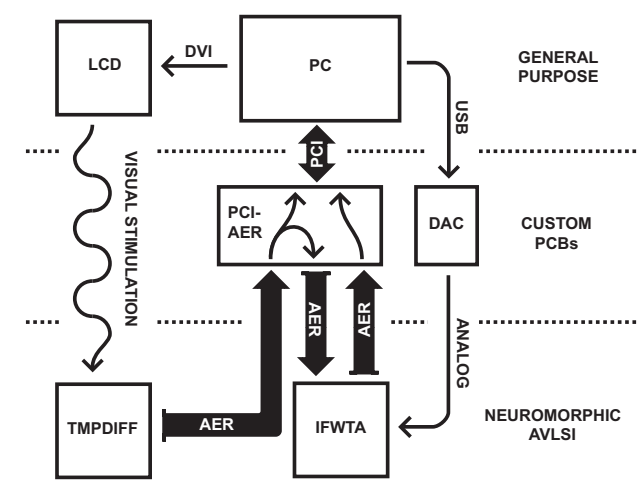

Fig. 1. AER vision system setup. The PCI-AER board routes output events of the TMPDIFF chip in response to visual stimuli to the IFWTA chip and monitors the activity of both chips. The PC controls the LCD screen for stimulus presentation, the PCI-AER board and the DAC board.

PCI bus of a host computer. It supports a real-time routing, programmable connectivity, and monitoring and stimulation of address events. Each PCI-AER board can host up to four sender and four receiver chips, and multiple PCI-AER boards can be shared on the PCI bus. The supporting hardware comprises a custom Digital to Analog Converter (DAC) board [9] for setting the analog biases of the neuromorphic chips, an LCD screen for presenting visual stimuli, and a workstation for hosting and controlling the PCI-AER board, programming the DAC board and controlling the LCD screen.

Even though the system described here comprises only two chips, it is can be easily extended to include multiple instances of the same IFWTA chip, or of analogous AER chips, using more ports of the PCI-AER board, or more PCI-AER boards.

\section{A. The TMPDIFF chip}

The TMPDIFF chip implements the sensing stage of our system. The chip produces asynchronous address-events in response to temporal changes in brightness. The stream of events encodes contrast changes rather than absolute illumination intensities. The retinal computation is optimized to deliver relevant information and to discard redundancy using high temporal and low spatial resolution, similar to the biological magnocellular pathway. As the TMPDIFF chip responds only to temporal changes in log intensity, static scenes produce no output. Image motion produces spike events that represent relative changes in image intensity. This operation in continuous form is represented mathematically by the following temporal relation on the pixel illumination $I$ :

$$
\frac{d}{d t} \log I=\frac{d I / d t}{I}
$$

This temporal derivative is self-normalized. By this normalization, the derivative encodes relative contrasts rather than absolute illumination differences. Contrasts are determined by differences in reflectance of objects independent of overall scene illumination. The events generated by TMPDIFF are changes in Eq. 1 that exceed a threshold and are $\mathrm{ON}$ or

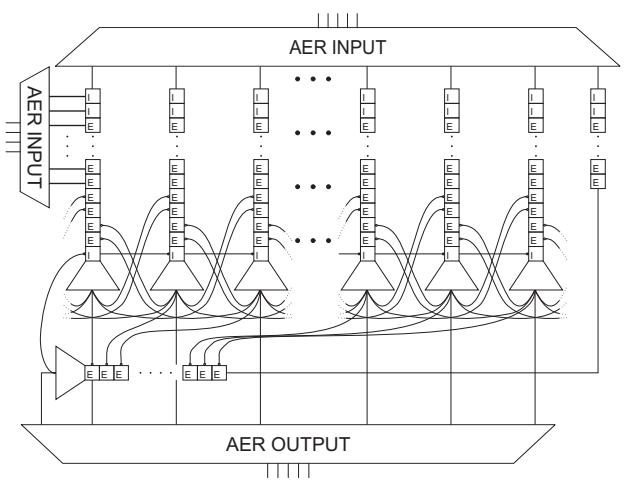

Fig. 2. Chip architecture. Squares represent excitatory (E) and inhibitory (I) synapses, trapezoids represent I\&F neurons. The I\&F neurons can transmit their spikes off-chip and/or to locally connected synapses (see text for details).

OFF type depending on the sign of the change since the last event. Pixel output consists of the stream of $\mathrm{ON}$ and OFF events. The imager, more thoroughly described in [10], [11], consists of an array of $32 \times 32$ pixels, a $y$-arbiter, an $x-$ arbiter and a common address bus with two encoders [12]. An event occurring in a pixel is communicated to the outside of the chip as an 11-bit address that encodes the pixel X-Y location and the polarity (ON or OFF) of the event. Events are processed asynchronously in order of their arrival time. In case of colliding events the latter are queued. The imager is a real-time device, which means that an event is communicated within 100ns of its occurrence. The AER communication system is particularly well suited for this application because it dedicates the full communication bandwidth to the active pixels of the imager and preserves timing information.

\section{B. The IFWTA chip}

The architecture of the IFWTA chip is shown in Fig. 2. It is a two-dimensional array containing a row of 32 Integrate-andFire (I\&F) neurons, each connected to a column of afferent synaptic circuits. Each column contains 14 AER excitatory synapses, 2 AER inhibitory synapses and 6 locally connected (hard-wired) synapses. When an address-event is received, the synapse with the corresponding row and column address is stimulated. If the address-events routed to the neuron integrate to the neuron's voltage threshold for spiking, then that neuron generates an address-event which is transmitted off-chip. The AER input synapses can be used to implement arbitrary network architectures, by (re)mapping address-events via the PCI-AER board.

Synapses with local hard-wired connectivity are used to realize a competitive soft winner-take-all (WTA) network with nearest neighbor and second nearest neighbor recurrent interactions (see Fig. 2 and Fig. 3): 31 neurons of the array send their spikes to 31 local excitatory synapses on the global inhibitory neuron; the inhibitory neuron, in turn, stimulates the local inhibitory synapses of the 31 excitatory neurons; each excitatory neuron stimulates its first and second neighbors on both sides using two sets of locally connected synapses. The first and second neighbor connections of the neurons at 


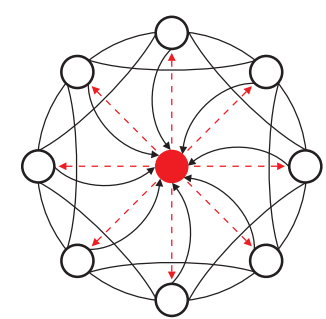

Fig. 3. Schematic representation of the connectivity pattern implemented by the internal hard-wired connections (closed boundary condition). Empty circles represent excitatory neurons and the filled circle represents the global inhibitory neuron. Solid/dashed lines represent excitatory/inhibitory connections. Connections with arrowheads are monodirectional, all the others are bidirectional.

the edges of the array are connected to pads. This allows us to leave the network open, or implement closed boundary conditions (to form a ring of neurons [13]), using off-chip jumpers.

All of the synapses on the chip can be switched off by appropriately setting the external bias voltages that control their synaptic weights. This allows us to inactivate either the local or the AER synaptic connections, or to use them in some arbitrary combination. A detailed description of the IFWTA chip was presented in [14].

\section{ORIENTATION SELECTIVITY EXPERIMENTS}

Orientation selectivity is achieved by appropriately mapping feed-forward connections from the TMPDIFF pixels to the IFWTA chip neurons (via the PCI-AER board), and by activating the local recurrent connections on the IFWTA chip. The feed-forward mapping is set so that each IFWTA neuron is excited by all the pixels of the TMPDIFF chip belonging to a central bar with a specific orientation. We implemented 31 sets of mapping tables that map 31 differently oriented bars onto the 31 excitatory neurons of the IFWTA chip.

In our experiments we displayed to the TMPDIFF chip flashing oriented white bars on a dark background using an LCD display. The activity of the TMPDIFF chip was monitored by the PCI-AER board and transmitted (via the PCI-AER board mapping tables) to the IFWTA chip. We time-stamped and logged both the TMPDIFF and IFWTA address-events for data analysis. To characterize the system we collected the system's activity in response to oriented flashing bars with 30 different orientations. Each flashing bar was displayed for approximately 40 seconds, flashing at a rate of about $3 \mathrm{~Hz}$. The monitoring of the address-event data lasted for 25 seconds, starting 5 seconds after stimulus onset. Figure 4 shows the integrated response of the TMPDIFF chip to the 30 orientations as grayscale images.

We repeated the same experiment for two different conditions, in terms of the local connectivity of the IFWTA chip. In the first condition the biases of the IFWTA chip were set to implement a purely feed-forward model: local recurrent synapses were inactive and the neurons' input was completely determined by the activity of the retinal pixels. Subsequently,

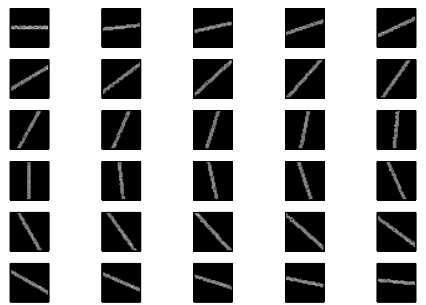

Fig. 4. Integrated response of the silicon retina to oriented flashing bars. Gray levels represent the average pixel activity ( $\mathrm{ON}$ events) over the monitoring period.

we activated the recurrent connectivity to implement the feedback model maintaining all other parameters unchanged. Three sets of local synapses were used: first neighbor excitatory to excitatory synapses to simulate the mutually excitatory connections among cells with similar preferred orientation, inhibitory and excitatory synapses connecting the global inhibitory neuron to the excitatory neurons and vice versa to simulate the mutual inhibition among cells with different preferred orientation.

Orientation tuning curves (i.e. graphs of neural response vs stimulus orientation) are typically measured in experiments related to the characterization of orientation selectivity in visual cortical neurons. We applied the same analysis to our data: the recorded activity of the IFWTA neurons was used to compute the mean firing rate of each neuron in response to the stimuli and tuning curves were obtained by plotting these data for each neuron as a function of orientation (see Fig. 5).

The TMPDIFF central pixels are mapped to all neurons, therefore each IFWTA neuron is also receiving input events when its non-preferred orientation is presented to the retina. The effect of this "base line" input is clearly visible in the feed-forward model, where the activity of the IFWTA neurons simply reflect the input from the retina. In this case, the frequencies in the tuning curves are greater than zero for all orientation and a maximum is observed at the preferred orientation. In the feed-back model the "base line" activity is suppressed and the activity in response to the preferred orientation is amplified.

We fitted the tuning curves to quantitatively estimate the effect of recurrent connectivity on the response of the orientation selective neurons. We used a von Mises function as fitting function [15], defined as

$$
M(\theta)=A e^{k[\cos 2(\theta-\phi)-1]}
$$

where $A$ is the value of the function at the preferred orientation $\phi$, and $k$ is a width parameter, from which the half-width at half-height $\theta_{0.5}$ may be calculated (in radians) as:

$$
\theta_{0.5}=0.5 \arccos [(\ln 0.5+k) / k] ; k>-0.5 \ln 0.5
$$

The von Mises function approximates a Gaussian in shape over a biologically likely range of values of $k$. A leastsquares fitting of the data to the von Mises function was used to estimate the parameters of the tuning curve of each 


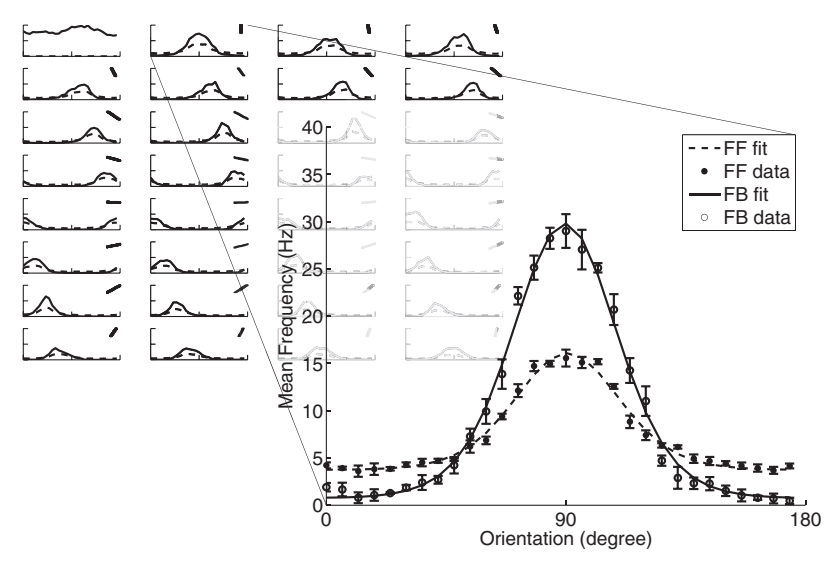

Fig. 5. Tuning curves for the feed-forward (dashed line) and the feed-back (solid line) model of orientation selectivity. The mean frequency $(\mathrm{Hz})$ of each neuron is plotted as a function of stimulus orientation. The top left graph shows the activity of the inhibitory neuron, the other graphs show the activity of the excitatory neurons (a bar representing the retinal pixels mapped to the neuron, i.e. its preferred orientation, is shown in each plot).

\begin{tabular}{|l|r|r|r|r|}
\cline { 2 - 5 } \multicolumn{1}{c|}{} & \multicolumn{2}{c|}{ Feed-forward Model } & \multicolumn{2}{c|}{ Feed-back Model } \\
\cline { 2 - 5 } \multicolumn{1}{c|}{} & Mean & \multicolumn{1}{c|}{ STD } & Mean & \multicolumn{1}{c|}{ STD } \\
\hline A (Hz) & 10 & 2 & 19 & 4 \\
\hline$\theta_{0.5}$ (degrees) & 21 & 2 & 19 & 2 \\
\hline Baseline activity $(\mathrm{Hz})$ & 1.7 & 0.6 & 0.07 & 0.11 \\
\hline $\begin{array}{l}\text { Preferred orientation } \\
\text { error (degrees) }\end{array}$ & 3 & 2 & 3 & 2 \\
\hline
\end{tabular}

TABLE I

MEAN AND STANDARD DEVIATION (STD) OVER THE POPULATION OF 31 ORIENTATION SELECTIVE NEURONS.

selective oriented neuron. The R-square value (the square of the correlation between the measured values and the values predicted by the fit) provides a measure of the goodness of the fit; it can take on any value between 0 and 1 , with a value closer to 1 indicating a better fit. The mean R-square across all fits is 0.982 and its standard deviation is $9 \times 10^{-3}$ : on average the fits explain $98 \%$ of the total variation in the data. The mean parameter values estimated from the fits are listed in Tab. I. This table confirms in a quantitative way what can be observed qualitatively in Fig.5: the network in the feedback configuration amplifies (with respect to the feed-forward configuration) the activity of the neurons at the preferred orientation. On average the peak activity in the feed-back network is twice the peak activity in the feed-forward network. Sharpening of the tuning curves is also observed: on average the ratio between the half-width at half-height for the two configurations is 0.9 (feed-back over feed-forward).

\section{CONCLUSION}

We have described a real-time multi-chip system consisting of a retina and a general AER competitive network composed of spiking neurons (the IFWTA chip) that can be used to implement in real time computational models of orientation tuning based on recurrent connectivity patterns. The VLSI neurons can detect the orientation of a visual stimulus, and have a response tuning similar to that observed in the mammalian visual cortex. The system is able to implement arbitrary mappings of spikes from the neuromorphic sensors to the IFWTA chip neurons, and strengths of the local recurrent connections on the IFWTA chip can also be modulated. This flexibility will allow us to explore further the range of collective processing possible in these circuits, and apply them to other tasks of feature detection in vision, and other modalities.

\section{ACKNOWLEDGMENT}

This work was supported by the ALAVLSI (IST-200138099), CAVIAR (IST-2001-34124), and DAISY (FP6-2005015803) EU grants, and by the ETH TH 0-20174-04 grant. We thank P. Del Giudice and V. Dante (ISS), for original design of the PCI-AER board and A. Whatley, G. Dietrich, S. Zahnd, M. Oster, F. Roth, and C. Girardin (INI), for help with the hardware setup and data analysis.

\section{REFERENCES}

[1] R. J. Douglas and K. A. C. Martin, "Neural circuits of the neocortex," Annual Review of Neuroscience, vol. 27, pp. 419-51, 2004.

[2] D. Ferster and K. D. Miller, "Neural mechanisms of orientation selectivity in the visual cortex," Anпи. Rev. Neurosci., vol. 23, pp. 441-71, 2000 .

[3] T. Serrano-Gotarredona, A. G. Andreou, and B. Linares-Barranco, "AER imager filtering architecture for vision-processing systems," IEEE Transaction on Circuits and Systems - I, vol. 46, pp. 1064-71, 1999.

[4] P. Venier, A. Mortara, X. Arreguit, and E. A. Vittoz, "An integrated cortical layer for orientation enhancement," IEEE Journal of Solid-State Circuits, vol. 32, no. 2, pp. 177-86, 1997.

[5] G. Cauwenberghs and J. Waskiewicz, "Focal-plane analog VLSI cellular implementation of the boundary contour system," IEEE Transaction on Circuits and Systems - I, vol. 46, no. 2, pp. 1064-71, 1999.

[6] S.-C. Liu, J. Kramer, G. Indiveri, T. Delbruck, T. Burg, and R. Douglas, "Orientation-selective aVLSI spiking neurons," Neural Networks, vol. 14, no. 6/7, pp. 629-643, 2001, special Issue on Spiking Neurons in Neuroscience and Technology.

[7] T. Y. W. Choi, P. A. Merolla, J. V. Arthur, K. A. Boahen, and B. E. Shi, "Neuromorphic implementation of orientation hypercolumns," IEEE Transactions on Circuits and Systems I, vol. 52, no. 6, pp. 1049-60, 2005.

[8] V. Dante, P. Del Giudice, and A. M. Whatley, "PCI-AER - hardware and software for interfacing to address-event based neuromorphic systems," in The Neuromorphic Engineer, 2005, vol. 2, no. 1, pp. 5-6.

[9] M. Oster, "Tuning aVLSI chips with a mouse click," The Neuromorphic Engineer, vol. 2, no. 1, p. 9, 2005.

[10] J. Kramer, "An integrated optical transient sensor," IEEE Trans. on Circuits and Systems II, vol. 49, no. 9, pp. 612-628, Sep 2002.

[11] P. Lichtsteiner, T. Delbruck, and J. Kramer, "Improved ON/OFF temporaly differentiating address-event imager," in 11th IEEE International Conference on Electronics, Circuits and Systems. IEEE, December 2004, pp. 211-214.

[12] K. Boahen, "Communicating neuronal ensembles between neuromorphic chips," in Neuromorphic Systems Engineering, T. S. Lande, Ed. Norwell, MA: Kluwer Academic, 1998, pp. 229-259.

[13] R. Hahnloser, R. Sarpeshkar, M. Mahowald, R. J. Douglas, and S. Seung, "Digital selection and analog amplification co-exist in an electronic circuit inspired by neocortex," Nature, vol. 405, no. 6789, pp. 947-951, 2000

[14] E. Chicca, G. Indiveri, and R. J. Douglas, "An event based VLSI network of integrate-and-fire neurons," in Proceedings of IEEE International Symposium on Circuits and Systems. IEEE, 2004, pp. V-357-V-360.

[15] N. V. Swindale, "Orientation tuning curves: Empirical description and estimation of parameters," Biological Cybernetics, vol. 78, pp. 45-56, 1998. 